ORIGINAL ARTICLE

\title{
Blood tests in tired elite athletes: expectations of athletes, coaches and sport science/sports medicine staff
}

\author{
K E Fallon
}

Br J Sports Med 2007;41:41-44. doi: 10.1136/bjsm.2006.030999

Background: The issue of the expectations of elite athletes, their coaches and non-medically qualified athlete support staff of consultations with sports physicians has not been previously dealt with in the sports medicine literature. As fulfilment of expectations of the content of a consultation may influence patient's satisfaction and clinical outcome, it is important to assess the expectations of athletes and, most importantly, coaches.

Objective: To assess the expectations and beliefs about fatigue, particularly in relation to blood tests, of athletes, their coaches and support staff in the specific context of tiredness of $<7$ days' duration.

Subjects: 28 senior sports science or non-medically qualified sports medicine staff, 22 elite coaches and 62 elite athletes from the Australian Institute of Sport were included in this study.

Methods: A single questionnaire.

Results: The expectation for a blood test at the initial consultation for short-term fatigue was particularly high among athletes (81\%) and coaches (91\%). This expectation increased in athletes if their performance was worsening. All groups unanimously suggested that a blood test be performed in cases of more prolonged

Correspondence to: Associate Professor K E Fallon, Department of Sports Medicine, Australian Institute of Sport, PO Box 176, Belconnen 2616, Canberra, Australia; fallonk@ausport.gov.au

Accepted 9 October 2006 fatigue. Increase in total training load was perceived to be the most important cause of fatigue, but issues relating to sleep were also thought to be highly relevant. All groups suggested that blood tests provide some degree of reassurance, and all groups suggested that the most important blood tests that might be performed related to exclusion of iron deficiency, anaemia and infection.

Conclusion: Athletes and their coaches generally expect that blood tests will be performed even when fatigue has been present for $<1$ week. This is at odds with currently available evidence of the diagnostic utility of these tests. Despite the current evidence base, individual factors in the athletes, coaches and doctors need to be considered when deciding on whether such testing has to be performed.

$\mathrm{T}$ he issue of the expectations of elite athletes, their coaches and non-medically qualified athlete support staff of consultations with sports physicians has not been dealt with previously in the sports medicine literature. As fulfilment of expectations of the content of a consultation may influence patient's satisfaction ${ }^{1}$ and clinical outcome, it is important to assess the expectations of the above-mentioned groups, particularly when their expectations may not be aligned with the evidence base for medical procedures such as ordering of investigations.

Several studies have assessed patients' expectations of history taking, clinical examination and ordering of tests as components of a consultation. In a questionnaire study of a group of patients attending an internal medicine practice, 50\% expected aspects of the clinical history to be considered, $61 \%$ a physical examination, $36 \%$ blood tests and $17 \% x$ rays or scans. ${ }^{2}$ In a primary care practice environment, both patients' desire, defined as "if a patient wanted a specific element of a consultation in the next year or two", and expectations, defined as "how likely patients thought it was that a doctor would order or recommend each element some time in the next year or two" were assessed. ${ }^{3}$ For a blood count, desire was rated $37-45 \%$ and expectation $22-42 \%$, and for aspects of the physical examination, desire was rated $67-86 \%$ and expectation $69-86 \%$. These figures are similar to those found by Peck et al, ${ }^{4}$ who noted that in $30 \%$ of consultations there was an expectation of a test of some variety.

Kravitz et $a l^{5}$ found that $18 \%$ of the patients of a sample of internal medicine doctors had one or more unfulfilled expectations, with physical examination (5.5\%), laboratory testing $(5.2 \%)$ and history taking $(4.9 \%)$ occupying three of the top four unmet expectations.
Presentation of elite athletes with fatigue or tiredness is not infrequent in a sports medicine practice. Anecdotal evidence indicates that some athletes and many coaches expect blood tests to be performed in this clinical situation. However, there is also recently published evidence that, particularly in cases where fatigue is of short duration ( $<3$ weeks), blood tests do not contribute to the diagnostic outcome. ${ }^{6}$

In view of this potential dichotomy and the potential consequences, this study aims to assess the expectations of athletes, their coaches and support staff, particularly in relation to blood tests, in the specific context of tiredness of $<7$ days' duration.

\section{METHODS}

Approval for this prospective study was obtained from the ethics committee of the Australian Institute of Sport (AIS). Thirty eight senior sports science or non-medically qualified sports medicine (SSSM) staff at the AIS, 24 AIS coaches and 62 elite athletes were approached by personal contact to participate in the study.

Those who gave their consent completed a three page questionnaire in which they were asked about expectations of the content of a consultation when an athlete presented with a complaint of fatigue or tiredness of 5-7 days' duration, important areas in the clinical history, types of blood test that might be performed, perceived causes of short-term fatigue and benefits of having a blood test if tests had been shown to be not useful in making a diagnosis of short-term fatigue. Each group was then asked if they would have been disappointed or felt

Abbreviations: AIS, Australian Institute of Sport; SSSM, sports science and sports medicine 
Table 1 Percentages of each group expecting a history to be taken, an examination to be performed, a blood test to be taken or a scan performed

\begin{tabular}{lccc}
\hline & Athletes & Coaches & SSSM staff \\
\hline History & & & \\
Yes & 98 & 95 & 100 \\
No & 2 & 0 & 0 \\
$\quad$ No answer & 0 & 5 & 0 \\
Examination & & & \\
$\quad$ Yes & 82 & 95 & 64 \\
$\quad$ No & 15 & 0 & 36 \\
$\quad$ No answer & 3 & 5 & 0 \\
Blood test & & & \\
Yes & 81 & 91 & 64 \\
$\quad$ No & 19 & 9 & 36 \\
$\quad$ No answer & 3 & 0 & 0 \\
Scan/x ray & & & \\
$\quad$ Yes & 0 & 5 & 0 \\
$\quad$ No & 97 & 41 & 100 \\
No answer & 3 & 54 & 0 \\
\hline
\end{tabular}

SSSM, sports science and sports medicine.

that a complete job had not been done if a blood test was not performed at the initial consultation, and similarly whether a blood test would be expected if performance was deteriorating or if the athlete had been fatigued for 4 weeks.

Athletes were asked to indicate their age, sex, sport and years involved in sport at the elite level (defined as state representation or above). Coaches were asked to indicate their highest coaching qualification and formal academic qualification. Sports science or non-medically qualified sports medicine staff were asked to indicate their professional group and highest academic qualification.

\section{RESULTS}

In all, $28(74 \%)$ of 38 senior sports science or non-medically qualified sports medicine staff responded to the survey. These included six exercise physiologists, four sport psychologists, six physiotherapists, four dietitians, four strength and conditioning specialists, two soft tissue therapists, one biomechanist and one who failed to nominate a profession. The highest academic qualification attained in this group was a $\mathrm{PhD}(8)$, a Master's degree (13), a Graduate diploma ${ }^{2}$ (2), a Bachelor's degree (5) or certificate from a college of advanced education (1).

In all, 22 (92\%) of 24 elite coaches responded to the survey. The highest academic qualification attained in this group was a Master's degree (2), Graduate diploma (9), Graduate certificate (1), Bachelor's degree (8), certificate from a college of advanced education (1) or completion of secondary school (1). The

Table 2 Rankings for each group based on most common responses for areas of importance in the clinical history

\begin{tabular}{llll}
\hline Item in history & Athletes & Coaches & SSSM staff \\
\hline Sleep characteristics & 1 & 4 & $=2$ \\
Training characteristics & 2 & 2 & 1 \\
(esp. load or changes) & & & \\
Diet & 3 & 5 & 4 \\
Specific symptoms & 4 & 6 & 8 \\
Characteristics of fatigue & 5 & 8 & 5 \\
Stress & 6 & 3 & 5 \\
Injury or illness & 6 & 7 & 2 \\
Recovery & 8 & NM & 6 \\
Previous episodes & 10 & 1 & 6 \\
Overall load & 10 & NM & 5 \\
\hline
\end{tabular}

NM, not mentioned; SSSM, sports science and sports medicine. highest coaching qualification obtained was level $4,{ }^{5}$ (the highest possible Australian qualification), level $3^{4}$ or level $2 .^{5}$

All the 62 athletes approached participated in the study. They represented rowing, track and field, swimming, archery, volleyball, basketball, football, skiing and netball. Their mean age was 21 years and the mean time of participation at the elite level was 6 years.

Table 1 shows the percentages of each group expecting a history to be taken, an examination to be performed, a blood test to be taken or a scan to be performed. The expectation for a blood test was particularly high among athletes and coaches.

Table 2 shows the rankings for each group based on the most common responses for areas of importance in the clinical history. NM indicates that this response was not mentioned by a particular group. As expected changes in training load or total training load were seen to be important in all groups. The importance of sleep characteristics was somewhat unexpected. This finding flags an area for further exploration.

Table 3 shows the rankings for each group for blood tests that might be ordered. All groups indicated that determination of iron levels was of prime importance and that exclusion of anaemia and infection was also important.

Table 4 shows the rankings for each group for perceptions of the utility of blood tests if they had been shown to be not useful in leading to a diagnosis. Reassurance of various forms and in relation to various problems was the most common answer for all groups.

Table 5 shows the rankings for each group for perceptions of the causes of short-term fatigue. While aspects of training figured most prominently, issues related to sleep and stress were also believed to be important.

Table 6 shows the percentage responses by group to expectations for blood tests in the case of (a) initial consultation for short-term fatigue, (b) deterioration in performance and (c) presence of tiredness for 4 weeks. The expectations for a blood test increase in athletes if their performance is worsening. All groups unanimously suggested that a blood test should be performed in cases of more prolonged fatigue.

\section{DISCUSSION}

As sports medicine matures and follows the more senior medical disciplines into the era of evidence-based medicine, the clouds of folklore and tradition, in which much of sports medicine and athletic training have been enveloped, will gradually clear. Much of this progress will require education of our major clients-athletes-and, perhaps more importantly, their coaches and ourselves. Collective understanding of the aims and objectives of our medical ministrations may even improve our outcomes. The issue of the alignment of knowledge, perceptions and practices of coaches is rarely dealt with, but the recent publication of a study in which beliefs and

Table 3 Rankings for each group for blood tests that might be ordered

\begin{tabular}{llll}
\hline Test & Athletes & Coaches & SSSM staff \\
\hline Iron levels & 1 & 1 & 1 \\
Anaemia & 2 & 2 & 4 \\
Infection & 2 & 5 & - \\
Glandular fever & 4 & 3 & 5 \\
Vitamins and minerals & 5 & - & - \\
No answer & 6 & 6 & - \\
White cell count & - & 4 & 2 \\
Full blood count & - & - & 3 \\
Liver function tests & - & - & 6 \\
\hline
\end{tabular}

SSSM, sports science and sports medicine. 
Table 4 Rankings for each group for perceptions of the utility of blood tests if they had been shown to be not useful in leading to a diagnosis

\begin{tabular}{llll}
\hline Benefit & Athletes & Coaches & SSSM staff \\
\hline Reassure that there is no serious problem & 1 & - & - \\
No answer & 1 & 1 & - \\
General reassurance/psychological & 3 & 3 & 1 \\
Reassure that iron level is OK & 4 & - & - \\
Reassure that diet is adequate & 5 & - & - \\
Excludes/diagnoses some problems & 5 & 2 & 2 \\
Tests general well-being & - & 3 & - \\
Convince athletes that they are OK & - & - & 3 \\
Placebo effect & - & - & 4 \\
\hline
\end{tabular}

practices of coaches related to stretching have been assessed ${ }^{8}$ threatens to open a gateway to this neglected field of study.

Despite what is known from general internal medicine about their relatively small contribution to diagnosis, blood tests assume an almost mystical importance in both sports and general medicine. This study explores how they might be better (or indeed less commonly) used in sports medicine.

In this study, coaches $(91 \%)$, athletes $(81 \%)$ and, to a lesser degree, sport science/medicine staff $(64 \%)$ indicated that a blood test should be performed at the first consultation for fatigue of short duration but, interestingly, when asked whether they would be disappointed or felt that a complete job had not been done at such a consultation, the corresponding figures were $68 \%, 32 \%$ and $46 \%$, respectively. Athletes' expectations were greater for blood tests if performance was worsening $(71 \%)$, and all groups felt that a blood test should be performed for more persistent fatigue.

If the evidence suggests that tests are not useful in diagnosis, at least in patients with short-term fatigue, ${ }^{67}$ why not just omit them? If only life was so simple. In making a decision about testing, doctor, athlete and coach factors all need to be considered. In treating elite athletes, the stakes are high and time pressure is constant-we cannot afford to miss a diagnosis. In the era of defensive medicine, tests are often performed "just in case" or because the unexpected may be disclosed, whereas in sports no doubt tests are performed for the same reasons. Just like other patients, athletes and coaches seek reassurance, and this study shows that reassurance of some sort is important to both coaches and athletes (table 4). But some of this reassurance cannot be simply provided by a blood test. The most common response of athletes to the question related to non-diagnostic benefits of a blood test was "reassure that there is no serious problem". Both coaches and SSSM staff noted "excludes/diagnoses some problems" as their second most common response. This is correct for some but not so for many problems. The coaches' third most common response was "tests general well-being"—clearly, blood tests do not do this. A normal blood test may not provide what some think that it does.

Reassurance by the performance of a blood test has been shown to be important to athletes, coaches and SSSM staff, but there is little consensus on when and whether investigations should be performed solely to reassure patients. ${ }^{9}$ It has been suggested that it is usually both unnecessary and unhelpful to investigate patients who have no organic pathology, ${ }^{10}$ but some patients may be difficult to reassure, and consideration needs to be given to personal and social factors, such as previous experiences, which may be obstacles to reassurance. ${ }^{11}$ Perhaps patient education can increase the chances of reassuring athletes and coaches, but the evidence for this is contradictory. One randomised controlled trial of lumbar spine $x$ ray versus an
Table 5 Rankings for each group for perceptions of the causes of short-term fatigue

\begin{tabular}{llll}
\hline Item in history & Athletes & Coaches & SSSM staff \\
\hline Sleep problems & 1 & 3 & 2 \\
Overtraining/increased training load & 2 & 1 & 1 \\
Incorrect diet & 3 & 4 & 4 \\
Poor or inadequate recovery & 4 & 6 & 5 \\
Stress & 5 & 2 & 3 \\
Illness & 6 & 5 & 6 \\
Training load & 7 & 5 & NM \\
\hline \multirow{2}{*}{ NM, not mentioned; SSSM, sports science and sports medicine. }
\end{tabular}

educational programme in patients with back pain and low risk for "red flag" conditions found no difference in resolution of symptoms, functional improvement or satisfaction between the groups. However, it should be noted that $31 \%$ of patients in the education group had received $x$ ray examination (mainly at other centres) by the conclusion of this study. ${ }^{12}$

But what about the effects of satisfaction of strong athlete and coach expectations? Firstly it must be realised that perceived patient pressure is a strong independent predictor of all doctors' behaviours ${ }^{13}$ but that doctors often do not correctly perceive patients' visit-specific expectations. ${ }^{1}$ To avoid truly unnecessary investigations, we need to check whether our assumptions of the patients' expectations are correct. Once that is established, meeting of patients' expectations may well be productive, but the evidence for the benefit of satisfaction of expectations related to tests is mixed.

In primary care settings, unmet expectations have been associated with decreased patient satisfaction, poor adherence and possibly worse outcomes. ${ }^{14}$ In a study in which, among other expectations, 54\% of patients had an expectation of a diagnostic test, those with no unmet expectations had less worry about serious illness and greater satisfaction and those who received diagnostic or prognostic information were more likely to have alleviation of symptoms and functional improvement at 2 weeks. ${ }^{15}$ However, when expectations for a test are studied in isolation, meeting such an expectation does not have a marked effect on satisfaction-the doctor's interpersonal behaviour is a more important predictor of satisfaction. ${ }^{16}$ So the question remains, should we fulfil the expectations of athletes and coaches by performing a blood test, in the hope that this will improve compliance with our therapeutic interventions?

Although the doctor's interpersonal behaviour may be an important predictor of patient satisfaction, a further issue that needs to be considered is the certainty with which doctors make

Table 6 Percentage responses by group to expectations for blood tests in the case of (a) initial consultation for short-term fatigue, (b) deterioration in performance and (c) presence of tiredness for 4 weeks

\begin{tabular}{lccll}
\hline Response (\%) & Yes & No & Maybe & No answer \\
\hline Initial consultation & 32 & 60 & 1 & 5 \\
$\quad$ Athletes & 68 & 32 & 0 & 0 \\
$\quad$ Coaches & 46 & 54 & 0 & 0 \\
$\quad$ SSSM staff & & & & \\
Performance worsening & & 24 & 5 & 0 \\
$\quad$ Athletes & 64 & 32 & 0 & 2 \\
$\quad$ Coaches & 46 & 54 & 0 & 0 \\
$\quad$ SSSM staff & & & & \\
$\quad$ Tired for four weeks & 100 & 0 & 0 & 0 \\
$\quad$ Athletes & 100 & 0 & 0 & 0 \\
$\quad$ Coaches & 100 & 0 & 0 & 0 \\
$\quad$ SSSM staff & & & & \\
\hline
\end{tabular}

SSSM, sports science and sports medicine. Yes indicates that a blood test is expected. 


\section{What is already known about this topic}

- The issue of the expectations of elite athletes, their coaches and non-medical athlete support staff of consultations with sports physicians has not been dealt with previously in the sports medicine literature.

\section{What this study adds}

- Athletes and their coaches generally expect that blood tests will be performed even when fatigue has been present for $<1$ week. Athletes' expectations increase markedly if performance is impaired by fatigue.

- All groups are unanimous in their expectation of a blood test in cases of fatigue lasting four weeks. Total training load or changes in training and sleep problems are the most commonly suggested causes of fatigue. All groups indicate that reassurance is provided by a blood test.

- Blood tests for iron levels, anaemia and infection are believed to be the most appropriate tests to be ordered by all groups.

provisional diagnoses. If it is possible, a firm provisional diagnosis should be made at the initial consultation, as it has been shown that unexplained complaints are positively related to ordering of tests as are patient's expectations that tests would be performed. ${ }^{17}$

Athletes, coaches and SSSM staff all ranked tests for iron levels as the most important tests for those presenting with fatigue. Tests for anaemia were ranked second by athletes and coaches and fourth by SSSM staff. Despite the fact that iron-related problems are relatively uncommon in elite athletes ${ }^{18}$ and were not found in a recent study of the clinical utility of blood tests in elite athletes with short-term fatigue, ${ }^{6}$ the prominence of these tests in the minds of athletes and coaches might assist in test selection if, all things being considered, a test is to be performed.

Finally, several issues related to the issue of tiredness and fatigue and not related to blood tests surfaced during this study. Of most interest was the prominent ranking of sleep problems when each group was asked about the causes of fatigue/tiredness (table 5). Sleep characteristics ranked first when athletes were asked about the aspects of the clinical history that they thought were important (table 2). Stress ranked high in the perceptions of coaches for both causes of fatigue (second) and important areas of clinical history (third). These areas and their interrelationship may be a fertile field for further research.

In summary, in relation to clinical situations where blood tests are believed not to be warranted, but where they might be expected, we have two options.

Firstly, we may take an evidence-based stand and attempt to educate both athletes and coaches at the time of consultation. Despite our best efforts, this approach is likely to leave lingering doubts in both groups.

Alternatively, we could

- prospectively educate our clientele about the utility of blood tests in sports medicine;

- assess the expectations of athletes and coaches of our consultations;

- meet reasonable expectations if we perceive that this will have clinical benefits;

- make a firm provisional diagnosis after our history and diagnosis;
- order a test if we estimate that more than verbal reassurance is required; and

- if a test is to be performed, in the scenario of short-term fatigue in an athlete, order only a haemoglobin and serum ferritin.

Perhaps the first option will suit the scientific mind of the doctor. I suspect that consideration of the second will better align our practice with the complex requirements and expectations of the athletes and coaches who are our primary patients.

\section{REFERENCES}

1 Rao JK, Weinberger M, Kroenke K. Visit specific expectations and patient centred outcomes. Arch Fam Med 2000;9:1 148-55.

2 Kravitz RL, Calahan EJ, Azari R, et al. Assessing patients' expectations in ambulatory medical practice. J Gen Intern Med 1997;12:67-72.

3 Zemencuk JK, Feighter JW, Hayward RA, et al. Patients' desires and expectations for medical care in primary care clinics. J Gen Intern Med $1998 ; 13: 273-6$

4 Peck BM, Ubel PA, Roter DL, et al. Do unmet expectations for specific test, referrals and new medication reduce patients' satisfactions? J Gen Intern Med, 2004; 19:1080-7.

5 Kravitz RL, Calahan EJ, Paterniti D, et al. Prevalence and sources of patients' unmet expectations for care. Ann Intern Med 1996;125:730-7.

6 Fallon K. Clinical utility of blood tests in elite athletes with short term fatigue. Br J Sports Med 2006;40:541-4.

7 Du Toit C, Locke S. The fatigued athlete: an audit of pathology test versus clinical diagnosis. J Sci Med Sport 2005;8(Suppl): 171.

8 Shehab R, Mirabelli M, Gorenflo D, et al. Pre-exercise stretching and sport related injuries: knowledge, attitudes and practices. Clin J Sports Med 2006;16:228-31.

9 Howard LM, Wessely S. Reappraising reassurance- the role of investigations. J Psychomatic Res 1996;41:307-11.

10 Howard I, Wessely S. Do investigations reassure patients with no organic pathology? Pract Neurol 2004;4:290-3.

11 Mc Donald IG, Daly J, Jelinek VM, et al. Opening Pandora's box: the unpredictability of reassurance by a normal test result. BMJ 1996;313:329-3.

12 Deyo RA, Diehl AK, Osenthal M. Reducing roentgenography use - can patient expectations be altered? Arch Int Med 1987;147:141-5.

13 Little P, Dorward M, Warner G, et al. Importance of patient pressure and perceived pressure and perceived medical need for investigation, referral, and prescribing in primary care: nested observational study. BMJ 2004;324:444-7.

14 Bell RA, Kravitz RL, Thom D, et al. Unmet expectations for care and the patientphysician relationship. J Gen Intern Med 2002;17:817-24.

15 Jackson JL, Kroenke K. The effect of unmet expectations among adults presenting with physical symptoms. Ann Intern Med 2001;134:889-97.

16 Froelich GW, Gilbert-Welch H. Meeting walk-in patients' expectations for testing - effects on satisfaction. J Gen Intern Med 1996;11:470-4.

17 van der Weijden T, van Velsen M, Dinant G-J, et al. Unexplained complaints in general practice: prevalence, patients' expectations and professionals' testordering behaviour. Med Decis Making 2003;23:226-31.

18 Fallon KE. Utility of haematological and iron-related screening in elite athletes. Clin J Sport Med 2004;14:145-52

\section{COMMENTARY}

This paper has a remarkable contemporary relevance and represents a common dilemma for sports physicians. The author cannot be faulted for his study design and selection of participants. The choice of clinical scenario was most appropriate. Unrealistic demands are often made of sports physicians when consulting with elite athletes. These issues are heightened at times of intense competition or during events such as Olympic and Commonwealth Games. All team physicians have experienced issues highlighted by this study which contributes to a sparse body of existing knowledge. It provides informed comment on athlete expectation, compliance and ultimate satisfaction. Educational strategies for patients are offered in a balanced manner.

D F Gerrard

University of Otago Medical School, Dunedin, New Zealand; david.gerrard@stonebow.otago.ac.nz 